\title{
Comparative sequence analysis of Solanum and Arabidopsis in a hot spot for pathogen resistance on potato chromosome $V$ reveals a patchwork of conserved and rapidly evolving genome segments Agim Ballvora*1, Anika Jöcker ${ }^{1}$, Prisca Viehöver ${ }^{1,2}$, Hirofumi Ishihara ${ }^{2,3}$, Jürgen Paal1', Khalid Meksem³ ${ }^{3}$ Rémy Bruggmann"4, Heiko Schoof ${ }^{1}$, Bernd Weisshaar ${ }^{1,2}$ and Christiane Gebhardt ${ }^{1}$
}

Address: ${ }^{1}$ Max-Planck Institut für Züchtungsforschung, Carl von Linné Weg 10, 50829 Köln, Germany, ${ }^{2}$ Institut für Genomforschung, Universität Bielefeld, Universitätsstrasse 25, 33615 Bielefeld, Germany, ${ }^{3}$ Southern Illinois University at Carbondale, Dept. of Plant, Soil and General Agriculture, Carbondale, IL62901-4415, USA and ${ }^{4}$ GSF Forschungszentrum für Umwelt und Gesundheit, Institut für Bioinformatik, Ingolstädter Landstr. 1, 85764 Neuherberg, Germany

Email: Agim Ballvora* - ballvora@mpiz-koeln.mpg.de; Anika Jöcker - joecker@mpiz-koeln.mpg.de; Prisca Viehöver - viehoeve@cebitec.unibielefeld.de; Hirofumi Ishihara - hishihar@cebitec.uni-bielefeld.de; Jürgen Paal - juergen.paal@altanapharma.com;

Khalid Meksem - meksemk@siu.edu; Rémy Bruggmann - remy.bruggmann@gsf.de; Heiko Schoof - schoof@mpiz-koeln.mpg.de;

Bernd Weisshaar - weisshaa@cebitec.uni-bielefeld.de; Christiane Gebhardt - gebhardt@mpiz-koeln.mpg.de

* Corresponding author

Published: 2 May 2007

BMC Genomics 2007, 8:1 12 doi:10.1186/147I-2164-8-II2
Received: 16 November 2006

Accepted: 2 May 2007

This article is available from: http://www.biomedcentral.com/I47I-2/64/8/II2

(c) 2007 Ballvora et al; licensee BioMed Central Ltd.

This is an Open Access article distributed under the terms of the Creative Commons Attribution License (http://creativecommons.org/licenses/by/2.0), which permits unrestricted use, distribution, and reproduction in any medium, provided the original work is properly cited.

\begin{abstract}
Background: Quantitative phenotypic variation of agronomic characters in crop plants is controlled by environmental and genetic factors (quantitative trait loci $=\mathrm{QTL}$ ). To understand the molecular basis of such QTL, the identification of the underlying genes is of primary interest and DNA sequence analysis of the genomic regions harboring QTL is a prerequisite for that. QTL mapping in potato (Solanum tuberosum) has identified a region on chromosome $\mathrm{V}$ tagged by DNA markers GP2I and GPI 79, which contains a number of important QTL, among others QTL for resistance to late blight caused by the oomycete Phytophthora infestans and to root cyst nematodes.
\end{abstract}

Results: To obtain genomic sequence for the targeted region on chromosome $\mathrm{V}$, two local BAC (bacterial artificial chromosome) contigs were constructed and sequenced, which corresponded to parts of the homologous chromosomes of the diploid, heterozygous genotype P6/2I0. Two contiguous sequences of 417,445 and 202,78 I base pairs were assembled and annotated. Gene-by-gene co-linearity was disrupted by non-allelic insertions of retrotransposon elements, stretches of diverged intergenic sequences, differences in gene content and gene order. The latter was caused by inversion of a $70 \mathrm{kbp}$ genomic fragment. These features were also found in comparison to orthologous sequence contigs from three homeologous chromosomes of Solanum demissum, a wild tuber bearing species. Functional annotation of the sequence identified 48 putative open reading frames (ORF) in one contig and 22 in the other, with an average of one ORF every $9 \mathrm{kbp}$. Ten ORFs were classified as resistance-gene-like, II as F-box-containing genes, I3 as transposable elements and three as transcription factors. Comparing potato to Arabidopsis thaliana annotated proteins revealed five micro-syntenic blocks of three to seven ORFs with A. thaliana chromosomes I, 3 and 5.

Conclusion: Comparative sequence analysis revealed highly conserved collinear regions that flank regions showing high variability and tandem duplicated genes. Sequence annotation revealed that the majority of the ORFs were members of multiple gene families. Comparing potato to Arabidopsis thaliana annotated proteins suggested fragmented structural conservation between these distantly related plant species. 


\section{Background}

The potato (Solanum tuberosum) is the most important crop of the Solanaceae. It is a tetraploid, non-inbred, annual plant species that is vegetatively propagated by tubers. Polyploidy and inbreeding depression prevent the generation of homozygous lines. When the ploidy level is reduced from $4 n$ to $2 n$, the diploid potatoes are self incompatible. Potato genotypes at all ploidy levels are therefore heterozygous [1]. The basic chromosome number of potato is twelve and its genome size is in the order of 800 to 1000 megabases, similar to the closely related tomato (Solanum lycopersicum). Detailed RFLP (restriction fragment length polymorphism) linkage maps have been constructed for the twelve chromosomes [2$5,63]$, which were subsequently used to locate in the potato genome factors controlling monogenic and polygenic traits of agronomic relevance such as resistance to pests and pathogens or tuber quality (e. g. starch and sugar content) (reviewed in $[1,6]$ ). When using the same locus specific DNA-based markers in different mapping populations, the positional information of the mapped factors controlling qualitative and quantitative traits can be compared and integrated. This comparison showed that a number of the factors which control qualitative $(R$ genes) or quantitative resistance $(\mathrm{QRL}=$ quantitative resistance loci) to different types of pathogens map to similar positions. These chromosomal regions are socalled hot-spots for pathogen resistance. One of the most conspicuous resistance hot-spots in the potato genome is located on potato chromosome $\mathrm{V}$, in a chromosome segment tagged by the DNA-based markers GP21 and GP179. The 3 cM interval between GP21 and GP179 [7] includes the $R$ genes $R \times 2$ and $N b$ both for resistance to Potato Virus $X[8,9]$ and the $R 1$ gene for race-specific resistance to the oomycete Phytophthora infestans causing late blight [10,7]. The same markers are also linked to QRL for P. infestans [11-14] and QRL for the root cyst nematodes G. rostochiensis and G. pallida [15-18]. As shown by QTL mapping [12$14,19,20]$, this region on potato chromosome $\mathrm{V}$ not only contains genes for resistance to various pathogens but also genes controlling plant vigor, plant maturity (the time the plant needs from planting to reach maturity under long day conditions), tuber yield, tuber starch and tuber sugar content.

Two $R$ genes from the chromosome $V$ resistance hot-spot have been functionally characterized, $R x 2$ for extreme resistance to Potato Virus $X[21]$ and $R 1$ for resistance to $P$. infestans [22]. Both $R$ genes are members of the superfamily of plant resistance genes characterized by a coiled coil (CC), a nucleotide binding (NB) and a leucine rich repeat (LRR) domain [23], but otherwise share low sequence similarity. $R 1$ has been introgressed from the allo-hexaploid - wild potato species Solanum demissum into the cultivated potato germplasm pool $[1,25]$ and is one member of a clustered gene family in the GP21GP179 interval $[22,25]$. The molecular basis of the QRL for late blight and root cyst nematodes in the same region is unknown. One possibility is that alleles of the R1 and/ or the $R x 2$ gene, or other members of the $R 1$ gene family and/or another resistance-gene-like (RGL) family in this genome region encode the factors for the quantitative resistance phenotypes, similar to classical plant genetic studies, where resistance loci with multiple specificities to different races of a pathogen may be alleles of the same gene, or tightly linked genes $[11,26]$. However, the resolution of QTL mapping in this region of the potato genome, even when based on large populations related by descent [27] is low. Genes physically linked to the R1 family, but structurally and functionally unrelated to $R 1$ or other RGLs, cannot be excluded as candidates for the QRL of interest. High resolution mapping and map-based cloning of QTL based on recombinant inbred populations or near isogenic lines [28] is not feasible in potato due to selfincompatibility. As an alternative approach, genomic sequencing and annotation can provide information on all putative genes present in the whole region, which then might be further examined for function as quantitative trait loci, first in silico by functional annotation and then experimentally by analysis of natural allelic diversity of positional candidates and complementation analysis using candidate gene allelic variants [28]. Powerful bioinformatic tools for gene annotation [29] and functional analysis of sequence related genes in model plants such as Arabidopsis thaliana and rice can facilitate the selection of functional candidate genes among all the positional candidates in a genome segment harboring QTL.

Parts of the genomic region corresponding to the GP21GP179 interval in S. demissum were sequenced and three different haplotypes $\mathrm{A}, \mathrm{B}$ and $\mathrm{C}$ equivalent to the three homeologous chromosome pairs of $S$. demissum were identified [25]. They demonstrated substantial structural variation among the haplotype sequences. In this paper, we report the genomic sequence analysis of two orthologous chromosome segments of the heterozygous, diploid Solanum tuberosum genotype P6/210 in the same GP21GP179 interval. Two independent bacterial artificial chromosome (BAC) contigs, corresponding to the homologous chromosomes of P6/210 were constructed, sequenced and annotated, thereby extending the genomic sequence information available in this functionally important region of the potato genome. Comparative sequence analysis revealed regions with severe structural distortions and deviations from gene by gene co-linearity, which are flanked by conserved regions showing microsynteny with the A. thaliana genome. 


\section{Results \\ Contig construction and BAC sequencing}

The potato clone P6/210 used to construct the BAC libraries was heterozygous for $R 1(R 1 / r 1)$. Physical mapping in the context of cloning the $R 1$ resistance gene had identified overlapping BAC insertions that originated from the homologous chromosomes carrying either the $R 1$ resistance allele or an $r 1$ allele for susceptibility [22]. In order to obtain two contigs, one for each homologous chromosome in the $R 1$ genomic region, the physical map was extended. Subsequently, we refer to the two contigs as the R1-contig and the r1-contig.

The R1-contig was extended proximally, starting from BA27c1 by clone BC93c12 (Figure 1). This BAC was identified by PCR screening of the BC library using specific primers that were developed based on the sequence of the proximal BAC end of BA27c1. Additional 34 BACs from this region, all containing one or more members of the R1 gene family, were identified by colony filter hybridization using as probe a $1.4 \mathrm{~kb}$ DNA fragment amplified with $R 1$ gene specific oligonucleotides 76-2sf2 and 76-2SR [22]. Among the clones containing R1 homologous genes were BA132h9 and BA213c14. Clone BA132h9 overlaps with BC93c12, and BA213c14 overlaps with BA87d17 (Figure 1). The gap between BA132h9 and BA87d17 was bridged by clone BC136j5 (Figure 1). From the seven BAC clones that constitute a minimal tiling path including the R1 locus, six BAC inserts were fully and one (BC93c12) was partially sequenced (Table 1 ). The r1-contig consisted of clones BA122p13 and BA76o11 and was extended proximally by clone BA111o5 (Figure 1). All three clones were fully sequenced (Table 1).

\section{Genomic sequence analysis}

The 620, 226 kbp genomic sequence obtained from seven $\mathrm{R} 1$ - and three $\mathrm{r} 1-\mathrm{BAC}$ insertions was assembled into two distinguished, unambiguous stretches of DNA sequence corresponding to the R1- and r1-contig of 417,445 base pairs [GenBank:EF514212] and 202,781 base pairs [Gen-

Table I: Summary of sequenced BAC insertions of genotype P6/ 210.

\begin{tabular}{|c|c|c|}
\hline BAC clone & Homologue & Sequence length [bp] \\
\hline BA47f2 & RI & 81,720 \\
\hline $\mathrm{BA} 27 \mathrm{cl}$ & RI & 91,301 \\
\hline $\mathrm{BC} 93 \mathrm{cl} 2$ & RI & 6,826 \\
\hline $\mathrm{BA} 132 \mathrm{~h} 9$ & RI & 74,715 \\
\hline $\mathrm{BCl} 36 \mathrm{j} 5$ & RI & 101,140 \\
\hline BA87dI7 & RI & 75,612 \\
\hline $\mathrm{BA} 2|3 \mathrm{c}| 4$ & RI & 72,505 \\
\hline BAI22p/3 & $\mathrm{rl}$ & 91,226 \\
\hline BA76oll & $\mathrm{rl}$ & 71,514 \\
\hline BAIIIo5 & $\mathrm{rl}$ & 76,593 \\
\hline
\end{tabular}

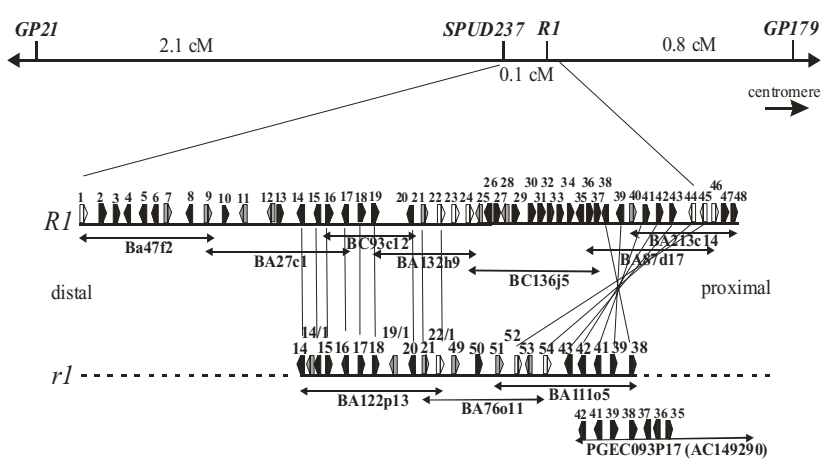

Figure I

Physical map and position of the RI- and rl-contig in the genetic interval GP2I-GPI 79 on potato chromosome $V$ and their annotated gene content. Annotated ORFs are shown as rectangles with arrowheads indicating the direction of transcription, and are numbered from I to 54 according to Table 2. ORFs showing sequence homology to RGLs are shown in white, putative polyproteins and transposon-like ORFs are shown in gray and all other gene models in black. Putative orthologs between the RI and rI contig are connected by thin lines. More detailed descriptions of the ORF annotation is provided in Additional file 5.

Bank:EF514213], respectively, using MegaMerger. Overall GC content in the R1- and r1-contig is $33.26 \%$ and $34.11 \%$, respectively.

The two contig sequences share highly similar and collinear regions, but these are interrupted by more variable regions (Figure 2). In addition, an inversion and an inverted repeat were identified. Tandem repeats corresponding to six copies of $R 1$ homologous genes in the R1 contig and three copies in the r1 contig were identified. These repeats are embedded in a region of non-alignable sequence.

For easier reference, we label regions along the R1 contig from A to F (Figure 2) based on features revealed by comparison of the R1 with the 1 contig using MUMmer (Figure 2). For region A, no sequence was obtained from r1. Region $\mathrm{B}$ is highly similar to the start of contig r1. In region $\mathrm{C}$, co-linearity and alignment are disturbed, and similarity is primarily detected in three tandem repeats. No similarity to $\mathrm{r} 1$ is detected in region $\mathrm{D}$. Region $\mathrm{E}$ $(69,850 \mathrm{bp})$ again aligns well with $\mathrm{r} 1$, but in reverse orientation, indicating a genomic inversion. Region $\mathrm{F}$, resembling region $\mathrm{C}$, is not aligned well but contains two tandem repeats that are highly similar to the tandem repeats in region $\mathrm{C}$, but in inverse orientation. Region $\mathrm{B}$ contains a palindromic structure discussed in more detail below. 


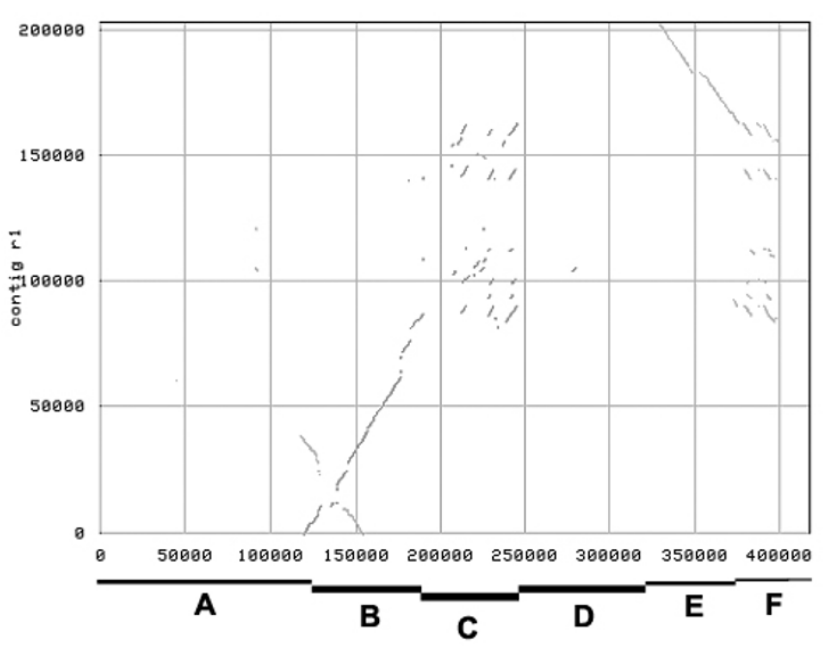

Figure 2

Dot-Plot comparison done using MUMmer of the DNA sequences of the RI- and rl-contig. Alignment between contigs starts at approximately $\mathrm{I} 20 \mathrm{kbp}$ in the RI-contig. The DNA sequence between approximately I $20 \mathrm{kbp}$ and $200 \mathrm{kbp}$ of the RI-contig (B) was highly similar to the rl-contig and included a palindromic structure of around $34 \mathrm{kbp}$. The regions from $200 \mathrm{kbp}$ to $245 \mathrm{kbp}$ (C) and again from 375 to $400 \mathrm{kbp}(\mathrm{F})$ of the $\mathrm{RI}$-contig contained conserved repeated sequences that were interrupted and flanked by sequences with low similarity between the two contigs. Segment D from 245 to $330 \mathrm{~kb}$ showed no similarity to the rl-contig. The segment from $330 \mathrm{kbp}$ to $400 \mathrm{kbp}(\mathrm{E})$ of the RI-contig was inverted relative to the $\mathrm{rl}$-contig.

Comparison to the A, B and C haplotypes of the orthologous genome region in Solanum demissum [25] revealed similar features (supplementary Fig. S3 and S4). The R1 contig is most closely related to the A sequence, with colinearity and high sequence identity (99\%). However, we orient BAC PGEC472P22 (accession AC151815) of A in reverse orientation compared to [25], thereby introducing the inversion of A and R1 relative to $\mathrm{r} 1$, B and C (see Discussion). The 1 contig is more similar to the sequences of $\mathrm{B}$ and $\mathrm{C}$ haplotypes of $S$. demissum.

Regions A and B show co-linearity with the S. demissum genomic regions II and III as defined by another study [25], whereas regions C through F correspond to S. demissum genomic regions IV and $\mathrm{V}$.

\section{Gene content}

We predict that the $\mathrm{R} 1$ contig contains 48 genes, seven of which are transposons and three are pseudogenes. Thirteen protein coding genes on the $\mathrm{r} 1$ contig can be identified as orthologs of collinear genes on R1. However, on R1 there are two additional protein coding genes. Six transposon genes are specific to $\mathrm{r} 1$, and for one pseudogene, we could not identify a partner on R1. On average, one ORF is annotated in every $9 \mathrm{kbp}$ of genomic sequence. The average GC content of exons is $39 \%$.

A putative function was assigned based on similarity to proteins of known function with an E-value smaller than $10^{-10}$ (Table 2, details in Additional file 5). Thirteen genes are related to retrotransposons. Two multigene families, Fbox proteins and NBS (NBS-LRR) type resistance genes, are represented by several members. Six proteins on contig R1 and three on $\mathrm{r} 1$ are members of the R1 gene family. At least eleven F-Box proteins are found on R1, but none of these are found in r1-contig.

Where sequence from both contigs was available, most proteins and some transposon-related genes are conserved between the R1 and $\mathrm{r} 1$ contig (Figure 1 and Additional file 5). Notable exceptions are 13 retroelements, six of which were found only in the $\mathrm{r} 1$ contig (ORF 14/1, ORF 19/1, ORF 21, ORF 49, ORF 51 and ORF 53) and 7 in the R1 contig (ORF 7, ORF 9, ORF 11, ORF 12, ORF 25, ORF 28 and ORF 40). The palindromic structure in region $\mathrm{B}$ is formed by an inverted repeat of two highly similar RNAdirected RNA polymerases (ORFs 14 and 16), separated by a hypothetical protein (ORF 15). The r1 specific retrotransposon 14/1 is inserted between the inverted repeats. Region E contains five genes that are conserved between $\mathrm{R} 1$ and $\mathrm{r} 1$ but in reverse order and orientation, indicating a genomic inversion. The proximal two genes are members of the $R 1$ family, ORF 44 being the functional $R 1$ resistance gene (accession AF447489) and the following ORF 45 is a tandem duplicate. ORFs 44 and 45 are conserved in sequence, but having inverse order and orientation, with resistance gene homologues 52 and 54 on contig $\mathrm{r} 1$, suggesting that the inversion includes these two genes. However, proteins 44 and 45 are more similar to each other than to 52 or 54 , indicating that they may have arisen through tandem duplication after the inversion event. Another resistance gene homologue (ORF 46) follows on contig R1, but is less similar to the other R1related genes. Also two genes (ORFs 47 and 48) could not be related to any locus on contig $\mathrm{r} 1$. This suggests that the proximal breakpoint of the genomic inversion in contig $\mathrm{R} 1$ is after gene 44 or 45 .

To map the distal breakpoint of the inversion event on contig R1, we used sequence from $S$. demissum BAC PGEC093P17 (accession $\underline{\mathrm{AC} 149290}$ ), as the sequence of contig r1 did not extend sufficiently far in proximal direction. The PGEC093P17 sequence contains orthologs of proteins 43, 42, 41, 39 and 38 in the same order and orientation as found on contig $\mathrm{r} 1$, therefore inverted with respect to $\mathrm{R} 1$. As in $\mathrm{r} 1$, the $\mathrm{R} 1$ specific transposon ORF 40 is not found in PGEC093P17 (Fig. 1). Proximal to protein 38 followed probable orthologs of proteins 37, 36 and 35 
Table 2: Genomic sequence annotation of the RI- and rl-contig.

\begin{tabular}{|c|c|c|c|}
\hline ORF No. (I) & Manual Functional Annotation (2) & ORF No.(I) & Manual Functional Annotation (2) \\
\hline I & Fragment of disease resistance protein & 27 & F-box protein-like \\
\hline 2 & ZF-HD homeobox protein & 28 & MuDR transposase \\
\hline 3 & Unknown protein & 29 & F-box protein \\
\hline 4 & Ribosomal protein L34e & 30 & F-box protein \\
\hline 5 & No apical meristem (NAM)-like & 31 & F-box protein \\
\hline 6 & Hypothetical protein & 32 & F-box protein \\
\hline 7 & Retrotransposon RNAse containing & 33 & F-box protein \\
\hline 8 & Hypothetical protein & 34 & F-box associated domain containing protein \\
\hline 9 & Retrotransposon & 35 & Hypothetical protein \\
\hline 10 & F-box protein & 36 & Pseudogene (F-box protein-like) \\
\hline 11 & Retrotransposon & 37 & F-box protein \\
\hline 12 & RNAseH containing reverse transcriptase & 38 & TCP transcription factor \\
\hline 13 & F-box protein & 39 & Unknown protein \\
\hline 14 & RNA dependent RNA polymerase & 40 & Retrotransposon \\
\hline $14 / 1$ & Transposon fragment & 41 & Unknown protein \\
\hline 15 & Hypothetical protein (Pseudogene) & 42 & Origin recognition complex subunit 6 \\
\hline 16 & RNA dependent RNA polymerase & 43 & Sterol desaturase/Acid phosphatase \\
\hline 17 & CAAX amino terminal protease & 44 & Disease resistance protein $(\mathrm{RI})$ \\
\hline 18 & Methyltransferase & 45 & Disease resistance protein (RI_I, $82 \%$ identity to $\mathrm{RI}$ ) \\
\hline 19 & Phytochrome kinase substrate & 46 & Disease resistance protein ( $R I \_2,49.3 \%$ identity to $\left.R I\right)$ \\
\hline $19 / 1$ & Retrotransposon & 47 & HVA22-like protein \\
\hline 20 & AAA ATPase & 48 & Regulator of chromosome condensation, $\mathrm{RCCl}$ \\
\hline 21 & Retrotransposon(Pseudogene) & 49 & Retrotransposon \\
\hline 22 & Disease resistance protein (RI-3, 74.7\% identity to $\mathrm{RI}$ ) & 50 & Hypothetical protein (Pseudogene) \\
\hline $22 / 1$ & Disease resistance protein (RI-6, $83.5 \%$ identity to $R I$ ) & 51 & Transposon-like \\
\hline 23 & Disease resistance protein ( $R I \_4,80.2 \%$ identity to $\left.R I\right)$ & 52 & Disease resistance protein ( $\mathrm{RI} 7$ 7, 80.7\% identity to $\mathrm{RI}$ ) \\
\hline 24 & Disease resistance protein (RI_5, 85.9\% to $\mathrm{RI})$ & 53 & Retrotransposon \\
\hline 25 & Retrotransposon & 54 & Disease resistance protein ( $\mathrm{RI}$-8, 83.2\% identity to $\mathrm{RI}$ ) \\
\hline 26 & F-box protein-like & & \\
\hline
\end{tabular}

(I)ORFs are numbered according to Figure I.

(2)Manual functional annotation was based on BLAST hits and/or domain prediction results, see Methods. More details are shown in Additional file 5.

in reverse order and orientation compared to contig R1, indicating that these three genes are part of the inversion. The remaining PGEC093P17 sequence proximal to ORF 35 contains only transposon fragments and hypothetical proteins, which are unrelated to genes distal to ORF 35 (mainly F-box genes, Table 2) in the R1-contig. This maps the distal inversion breakpoint in contig R1 between genes 34 and 35. As consequence of the inversion, genes 23 to 34 in the R1 contig are probably part of a region that is, at least at this genomic location, $\mathrm{R} 1$ specific. This region includes two R1 homologous genes (ORF 23 and 24) in tandem orientation to ORF 22, two transposon-related genes and a series of eight F-box genes. For the flanking regions of contig R1, we found almost perfect gene-bygene co-linearity to $\mathrm{r} 1$ or PGEC093P17. We could not assign three genes in contig $\mathrm{r} 1$ to any collinear region, two of which are transposons and one resembles a fragmented resistance gene.

\section{Microsynteny with the A. thaliana genome}

Five microsyntenic blocks were identified in A. thaliana based on the arbitrary criterion of finding at least three pairs of homologous protein sequences within a compa- rable physical distance (Table 3 and Additional file 6). When the potato annotated proteins were compared to the A. thaliana annotated proteins, only ORFs $6,8,15$ and 35 did not have any hit. Each member of the 'resistancegene-like' and 'F-box containing' gene families had more than 30 hits. Due to the high copy number, these two multigene families and the retrotransposons were not considered when searching for groups of in both species physically closely linked homologue pairs (microsynteny). The 26 remaining potato ORFs used for microsynteny analysis had on average 9.2 hits in the A. thaliana genome. The five syntenic blocks involve three sections within the potato contigs, (i) the distal ORFs 2, 3, 4 and 5, (ii) ORFs 17, 18 and 19 in the middle part and (iii) ORFs 38 to 48 in the proximal region. The syntenic blocks I, II and III comprise between $215 \mathrm{kbp}$ and $405 \mathrm{kbp}$ potato sequence and are syntenic with three different, smaller $A$. thaliana genome fragments from $7 \mathrm{kbp}$ to $54 \mathrm{kbp}$ on chromosome 1 . The four consecutive A. thaliana genes in block I have the same relative orientation of transcription and the same order as the homologous potato ORF 17, 19, 43 and 41 in the r1-contig (Table 3, Figure 1). The largest syntenic region identified in both species is block III, includ- 
ing 7 potato ORFs, which spans nearly the whole R1contig (405 kbp). The corresponding A. thaliana genome fragment is $54 \mathrm{kbp}$ in size and contains 12 annotated genes, seven in the same order and homologous to ORFs in the r1-contig, one F-box gene (At1G69630.1) and four other, consecutive genes that are not syntenic (AtG69650.1 to AtG69680.1). Block II with four syntenic ORFs is second in size (345 kbp). The related A. thaliana genome fragment of only $18 \mathrm{kbp}$ contain five annotated genes, four homologous to the potato ORFs and the fifth one different (AtG26860.1). Potato ORFs 2, 4 and 5 (block IV) and 2, 3 and 5 (block V) are located within 25 kbp in the R1-contig. The A. thaliana genome fragment IV on chromosome 3 is larger (106 kbp), whereas fragment $\mathrm{V}$ on chromosome 5 is nearly identical in size (28 kbp). The three ORFs in block $\mathrm{V}$ are not only located in genomic fragments of similar size in both species but also in the same order and in the same orientation of transcription. It is unlikely that the five syntenic blocks occur by chance only. We estimate the probability of finding three hits within $100 \mathrm{~kb}$ in $A$. thaliana to be less than $10^{-3}$ using an approximation described in [4]: $\mathrm{h}^{\mathrm{n}} \times(0.1 \mathrm{Mbp} / 121$ $\mathrm{Mbp})^{\mathrm{n}-1}$, where $\mathrm{h}$ is the average number of hits of the potato genes in A. thaliana (9.2), $\mathrm{n}$ the number of genes conserved in the syntenic block (3), $0.1 \mathrm{Mbp}$ the size of the syntenic block in $A$. thaliana and $121 \mathrm{Mbp}$ the size of the $A$. thaliana genome (giving $5 \times 10^{-4}$ for three genes with an average of 9.2 hits). None of the syntenic $A$. thaliana genome fragments include a putative or functional gene for pathogen resistance and only one includes an F- box containing gene. The A. thaliana gene RPP13 conferring resistance to Peronospora parasitica and having the highest sequence similarity to the $R 1$ gene family (Additional file 5) is located on chromosome 3 outside any putatively syntenic region.

\section{Discussion \\ Genome structure}

We found extensive co-linearity of protein-coding genes, interrupted by unilateral insertions of retrotransposons and a region of highly diverged DNA sequence in the vicinity of clusters of tandem duplicated genes. This corresponds to findings in the orthologous genomic region of hexaploid S. demissum [25]. We show that a $70 \mathrm{~kb}$ region containing ten protein-coding genes is inverted in both the R1 contig and presumably also the A haplotype of $S$. demissum. While sequence similarity at the nucleotide level was not sufficient to precisely map the inversion breakpoints in the intergenic regions, order and orientation of homologous gene pairs were consistent without exception and allowed to estimate the position of the inversion breakpoints. This inversion is not evident in the available sequence of the S. demissum A haplotype, as BAC PGEC472P22 (accession AC151815) mainly contains genes from the inversion and only few beyond the inversion breakpoint. The gap in the sequence of haplotype A presumably led to BAC PGEC472P22 being oriented to achieve co-linearity with $\mathrm{B}$ and $\mathrm{C}$ haplotypes. Kuang et al. [25] do not present any data, e.g. mapping of BAC ends or overlaps with neighboring BAC clones, to verify the orien-

Table 3: Syntenic blocks between potato annotated genes in the $\mathrm{RI}$ - and $\mathrm{rl}$-contigs and sequence related genes of $A$. thaliana.

\begin{tabular}{|c|c|c|c|c|c|c|}
\hline Syntenic block & S. tuberosum ORF & A. thaliana ORF (I) & A. thaliana BAC & $\begin{array}{l}\text { A. thaliana ORF position } \\
\text { (I) }[\mathrm{MbP}]\end{array}$ & A. thaliana block size & S. tuberosum block size \\
\hline \multirow[t]{4}{*}{1} & ORFI7 & AtIG I4270.I & FI4LI7 & 4.875 & $7 \mathrm{kbp}$ & $215 \mathrm{kbp}$ \\
\hline & ORFI9 & AtIGI4280.I & FI4LI7 & 4.878 & & \\
\hline & ORF43 & AtIGI4290.I & FI4LI7 & 4.880 & & \\
\hline & ORF4I & AtIGI4300.I & FI4LI7 & 4.882 & & \\
\hline \multirow[t]{4}{*}{ II } & ORF4 & AtIG26880.I & T2PII & 9.316 & 18 kbp & $345 \mathrm{kbp}$ \\
\hline & ORF5 & AtIG26870.I & T2PI I & 9.313 & & \\
\hline & ORFI8 & AtIG26850.I & T2PII & 9.301 & & \\
\hline & ORF42 & AtIG26840.I & T2PII & 9.298 & & \\
\hline \multirow[t]{7}{*}{ III } & ORF2 & AtIG69600.I & F24JI & 26.168 & 54 kbp & 405 kbp \\
\hline & ORF3 & AtIG69610.1 & T6C23 & 26.190 & & \\
\hline & ORF4 & AtIG69620.I & $\mathrm{T} 6 \mathrm{C} 23$ & 26.193 & & \\
\hline & ORF43 & AtIG69640.I & $\mathrm{T} 6 \mathrm{C} 23$ & 26.197 & & \\
\hline & ORF38 & AtIG69690.I & T6C23 & 26.221 & & \\
\hline & ORF47 & AtIG69700.I & T6C23 & 26.224 & & \\
\hline & ORF48 & AtIG69710.I & T6C23 & 26.226 & & \\
\hline \multirow[t]{3}{*}{ IV } & ORF2 & At3G28920.I & MYII 3 & $10.94 \mid$ & 106 kbp & $25 \mathrm{kbp}$ \\
\hline & ORF4 & At3G28900.I & K5K 13 & 10.904 & & \\
\hline & ORF5 & At3G29035.I & K5K 13 & I I.035 & & \\
\hline \multirow[t]{3}{*}{ V } & ORF2 & At5G39760.I & MKM2I & 15.928 & 28 kbp & 25 kbp \\
\hline & ORF3 & At5G39785.I & MKM2I & 15.946 & & \\
\hline & ORF5 & At5G39820.I & MKM2I & 15.956 & & \\
\hline
\end{tabular}

(1)A. thaliana gene identifiers and position coordinates on the AGI physical map in Megabasesare according to TAIR release 6. 
tation. Thus, we take the high sequence similarity, uninterrupted across the presumed inversion break point, between the R1 contig and PGEC472P22 to indicate that the inversion also exists in the A haplotype (Additional files 3 and 4).

Currently, there are only few examples where comparative structural analysis of orthologous genome segments was performed in crop plants over several hundred $\mathrm{kb}$. In all cases reported, micro-structural diversity was found, e.g. in Zea mays [30,31]. In maize the major contribution to diversity stems from LTR retrotransposons. In our analysis transposon related genes are less conserved, also suggesting recent insertions and deletions. In tomato ( $S$. lycopersicum), a segment on chromosome 6 containing the Mi-1 gene for resistance to root knot nematodes, which has been introgressed from $S$. peruvianum, was shown to be inverted in resistant when compared to susceptible tomato genotypes [32]. The situation at the potato $R 1$ locus described here remarkably resembles this finding. The R1-contig is part of a genome fragment of unknown size introgressed from S. demissum into S. tuberosum, whereas the r1-contig originated either from $S$. tuberosum or S. spegazzinii, another closely related tuber bearing Solanum species. P40, the parental donor of the $r 1$ allele, was an inter-specific hybrid between $S$. tuberosum and S. spegazzinii [33]. The structural differences between the homologous chromosomes could interfere with chromosome pairing and crossing-over during meiosis, explaining the low frequency of recombination observed in this region [22]. Similarly, in regions on tomato chromosome 6 and 11, where the resistance loci Mi and Tm2a, respectively, have been introgressed from the wild species $S$. peruvianum, a high degree of recombination suppression was observed $[32,34]$.

In the $\mathrm{R} 1$ contig, the inversion seems to have separated the $R 1$ resistance gene from a tandem array of $\mathrm{R} 1$ homologs (proteins 22, 23 and 24). We attempted to date the inversion relative to the duplications of tandem resistance genes by phylogenetic analysis of the protein sequences, but the results were inconclusive (data not shown). Proteins 23, 24, 44 and 45 clustered together. Proteins 22 and $22 / 1$ also clustered together, and even though gene 22 on the $\mathrm{R} 1$ contig is truncated at the N-terminus relative to 22/ 1 and the other R1 homologous proteins, we assume these to be an orthologous pair. For the other R1 homologues, allelic relationships are not clear, and they may have arisen through duplication after the divergence of R1 and r1.

Kuang et al [25] analyzed the same genomic region on potato chromosome $\mathrm{V}$ between three homeologous chromosomes of the allo-hexaploid potato species $S$. demissum. Alignment of the sequenced $S$. demissum BACs with the R1- and r1-contig identified haplotypes A and B/C as most similar but not identical to the R1- and r1-contig, respectively. Similarity of individual homologous protein pairs between $\mathrm{B}$ and $\mathrm{r} 1$ or $\mathrm{C}$ and $\mathrm{r} 1$ ranges between 90 and $99 \%$ identity on the amino acid level, with most, but not all proteins slightly more similar between $\mathrm{C}$ and $\mathrm{r} 1$ than between $\mathrm{B}$ and $\mathrm{r} 1$. On the other hand, the $\mathrm{B}$ haplotype is structurally more similar to $\mathrm{r} 1$, as $\mathrm{C}$ shows no tandem repeated $R 1$ homologous genes but only one single copy.

Remarkable is the discovery of highly conserved and seemingly rapidly evolving genome regions in close vicinity. The distinguishing feature, aside from the observed breakdown of nucleotide sequence similarity in non-coding regions and the lack of gene-by-gene co-linearity, is the presence of tandem repeated genes, namely $R 1$ homologs and F-box containing genes. Such tandem arrays have been found in other hypervariable genome regions [35] and may have lead to a greatly enhanced rate of evolution due to relaxed selection pressure on duplicated genes. Neighboring unique sequences are, in contrast, highly conserved. In the variable region, the R1 and r1 contigs and the three $S$. demissum haplotypes show striking structural variation, ranging from lack of the variable region in $S$. demissum haplotype $\mathrm{C}$ to the expanded set of F-box proteins found in the R1 contig. The latter are missing from $\mathrm{r} 1$ and $\mathrm{B}$. Instead, in the B haplotype the R1 homologous gene tandem array is more expanded (figure 3).

\section{Encoded proteins}

We found a gene density of one gene every $9 \mathrm{~kb}$, which is similar to previous findings in $S$. demissum $(7.6 \mathrm{~kb},[24])$ and tomato $(8 \mathrm{~kb},[36,37])$, but lower than $A$. thaliana (5 $\mathrm{kb},[38])$ and rice (6 kbp, [39]), and higher than in barley,

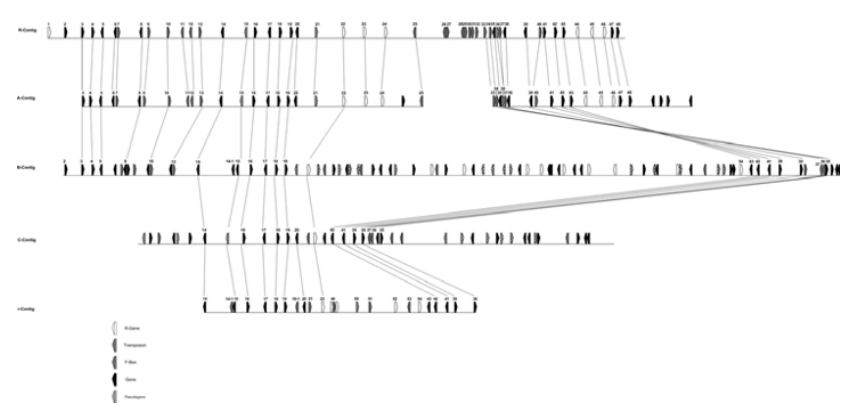

\section{Figure 3}

Comparison between gene loci of the RI- and rI contig as well as of the A, B and C haplotype of S. demissum. White polygons symbolize R-genes. Striped polygons indicate pseudogenes. Chequered polygons represent F-box genes. Gray polygons stand for transposons and all other genes are shown as black polygons. Orthologous genes are connected by black lines. 
where only three genes were found in a stretch of $60 \mathrm{kbp}$ genomic DNA [40]. The overall GC content was $37 \%$ and $39.5 \%$ within the putative gene coding regions. These values are comparable to tomato $37 \%$ overall GC content and $42 \%$ in coding regions, [36]) and A. thaliana (36\% overall GC content and $44 \%$ in coding regions, [38]) but lower than in rice (44\% overall GC content and 54\% in coding regions, [39]) and maize (47\% overall GC content and $55 \%$ in coding regions.

\section{The $R$ I gene family and disease resistance QTL}

Annotation of the R1 gene family in S. demissum haplotype A [25] and in the R1-contig was comparable but not identical. In the R1-contig, six putative full-length R1 homologous genes and no partial homolog were annotated besides the $R 1$ resistance gene itself. The six members of the $R 1$ gene family were organized in two clusters of three genes each (proteins 22, 23, 24 and $44=R 1,45$, $46)$. In the corresponding region of $S$. demissum haplotype $\mathrm{B}$, four putative full length $R 1$ homologous genes and six partial homologues were identified. In $S$. demissum haplotype C, one complete R1 homologue was found [24], whereas three complete members of the $R 1$ gene family (proteins 22/1, 52 and 54) were annotated in contig r1(Figures 1 and 3). Allelic relationships between the R1 homologues could not be deduced with certainty, but proteins 22 and 22/1 might be allelic based on collinear positions in the R1- and r1-contig, whereas proteins 44 and 45 might be allelic to 54 and 52 respectively as they are collinear under the assumption that they are part of the proposed genomic inversion.

Most of the molecular characterized plant $R$ genes are members of tightly linked gene families [41]. The $R 1$ gene family is no exception in this respect. Allelic variants of the nine identified members of the $R 1$ family or additional paralogous members that are not present in genotype P6/210 are non-exclusive candidates for the quantitative resistance traits in the resistance hot spot on potato chromosome V. At this point, we only know that some of the R1 homologues likely have functions other than the $R 1$ resistance gene. This is based on the observation that the $R 1$ homologue encoded by ORF 45 was not capable to complement the $R 1$ race specific resistance phenotype (A. B. unpublished results).

Genes sequence-related to retrotransposons, ribosomal genes, RNA dependent RNA polymerase and pseudogenes are ranked low for being functional candidates for the quantitative traits in this region of the potato genome. The remaining 30 putative genes, including hypothetical genes and genes with unknown function, are all positional candidates for the QTL. Of particular interest as new candidates for quantitative resistance loci, besides the members of the $R 1$ gene family, are the members of the F- box domain family. F-box proteins are involved in various signaling pathways in A. thaliana, and recently, F-box proteins are suggested to function as receptors for various plant hormones [42]. Furthermore, an F-box domain was identified in the SGT1 protein that was shown to play a role as co-chaperon in the stabilization of R-proteins $[43,44]$. With the annotation of the sequenced region, the list of positional candidate genes for the QTL is certainly not complete, as the sequence covers only part of the GP21-GP179 interval. To ultimately validate the role of any candidate gene for a QTL, complementation analysis with allelic variants is required. Unless high-throughput methods for complementation analysis become available, strategies to reduce the number of candidate genes to be considered for complementation analysis are necessary. For example, we perform functional testing by expression studies and by down-regulation of candidate gene expression by antisense or RNAi approaches [45]. The model plant $A$. thaliana may also be used to study the function of genes that are most closely sequence-related to potato positional candidate genes. Unfortunately, this approach may not be applicable to the F-box family, as this is also a highly expanded gene family in A. thaliana with diverse cellular roles.

\section{Synteny with A. thaliana}

We identified at least five microsyntenic relationships between the R1 contig and A. thaliana. These cover varying stretches of the genome, ranging from just four consecutive genes within $7 \mathrm{~kb}$ of $A$. thaliana and $25 \mathrm{~kb}$ of potato to basically the entire R1 contig, covering $405 \mathrm{~kb}$. Frequent insertion-deletion events can be detected. Similar patterns of interrupted co-linearity on the DNA sequence level were found among cereals [46] and between $A$. thaliana and rice [47]. In the highly collinear tomato genome $[2,5]$, genomic sequences of $57 \mathrm{kbp}$ and $106 \mathrm{kbp}$ on chromosome 2 [48] and 7 [37], respectively, have been compared to the $A$. thaliana genomic sequence. These studies revealed syntenic blocks of comparable redundancy and size with respect to the $A$. thaliana syntenic regions. In contrast to these previous studies, the contiguous potato sequence compared was 4 to 8 -times longer. This revealed that the syntenic potato genes in the R1-contig were organized in three clusters (ORF 2 to 5, ORF 17 to 19 and ORF 38 to 48) that were separated by two non-syntenic regions (ORF 6 to 16 and ORF 20 to 37). In the r1-contig, a non-syntenic region (ORF 20 to 54) separated two syntenic regions (ORF 17 to 19 and ORF 38 to 43 ).

The most notable of the syntenic relationships spans almost the complete R1 contig (405 kbp) and $54 \mathrm{kbp}$ of A. thaliana chromosome 1 . Seven genes are conserved in sequence, order and orientation, except for two from region $\mathrm{E}$ that show reverse order and orientation compared to $A$. thaliana. This could indicate that the genomic 
inversion occurred in the $\mathrm{R} 1$ lineage after the divergence of A. thaliana and potato, with $\mathrm{r} 1$ and S. demissum B and C haplotypes showing the ancestral orientation. In the R1 contig, a large number (18) of genes do not show synteny, whereas in the A. thaliana region this only applies to five genes. The discrepancy is less pronounced if the 17 tandemly duplicated genes in potato are ignored.

The non-syntenic regions correspond to the highly divergent regions between $\mathrm{R} 1$ and $\mathrm{r} 1$ and included all but one (ORF 40) transposon sequences, all F-box-containing genes and six of the ten resistance-gene-homologues. The annotation of the A. thaliana syntenic regions identified, besides the sequence related ORFs, some transposon sequences but only one F-box-containing gene and no resistance gene homolog. Moreover, the non-syntenic regions in the R1- and $\mathrm{r} 1$-contigs coincided with regions II and IV in S. demissum, which showed the highest divergence between the homeologous chromosome segments A, B and C [25]. This suggests that the genome of potato and related species in the sequenced region consists of a patchwork of faster and more slowly evolving segments.

The sequenced potato genomic segment covers a genetic distance of only 0.1 Centimorgan. At a hundred times larger scale, when genome-wide genetic maps of potato, sunflower, sugar beet and Prunus were compared to the A. thaliana physical map (macrosynteny), syntenic blocks from 1 to 20 Centimorgans were identified. A common fraction of the genomes of these distantly related plant species appear to have been conserved throughout the evolution of the dicots, when compared to the rest of the genome [49]. The GP21-GP179 interval was not part of a macrosyntenic block between potato and A. thaliana [4].

\section{Conclusion}

Two contiguous sequences of 417,445 and 202,781 base pairs were assembled and annotated for a region on potato chromosome $\mathrm{V}$, which contains genes controling several agronomic traits. Comparative sequence analysis revealed highly conserved collinear regions that flank regions showing high variability and tandem duplicated genes. The co-linearity between the homologous chromosomes was disrupted by non-allelic insertions of retrotransposon elements, stretches of diverged intergenic sequences, differences in gene content and gene order. The latter was mainly caused by inversion of a $70 \mathrm{kbp}$ genomic fragment.

Annotation of the genomic sequence identified 48 putative open reading frames (ORF) in one contig and 22 in the other, with an average of one ORF every $9 \mathrm{kbp}$. The majority of the ORFs were members of multiple gene families. Ten ORFs were classified as resistance-gene-like, 11 as F-box-containing genes, 13 as transposable elements and three as transcription factors. Comparing potato to Arabidopsis thaliana annotated proteins revealed five micro-syntenic blocks of three to seven ORFs with $A$. thaliana chromosomes 1, 3 and 5, suggesting fragmented structural conservation between these distantly related plant species.

\section{Methods \\ BAC-libraries}

For contig construction, two BAC genomic libraries were used, each consisting of ca. 100000 clones (two hundred and sixty four 384-well microtiter plates). Both libraries were generated from high molecular weight DNA of the diploid, heterozygous potato clone P6/210, a F1 hybrid of the parental clones P41 (H79.1506/1) and P40 (H80.696/4) [9]. The 'BA' library has been described [22]. The library ' $\mathrm{BC}$ ' was constructed in the cloning vector pBeloBAC11 [50] from partially EcoRI digested genomic DNA. The procedures for the construction of recombinant BAC clones, clone picking and storing were as described previously $[22,51]$. The average insertion size of the ' $\mathrm{BC}$ ' library was $80 \mathrm{kbp}$, corresponding to an, on average, 8fold coverage of the potato genome.

\section{BAC plasmid DNA isolation}

A single colony was pre-cultured in $250 \mu \mathrm{LB}$ medium including $12.5 \mathrm{mg} / \mathrm{l}$ tetracycline for clones from the "BA" library and $12.5 \mathrm{mg} / \mathrm{l}$ chloramphenicol for clones from the "BC" library. The pre-culture was used to inoculate 50 $\mathrm{ml}$ LB medium containing the corresponding antibiotic. Plasmid DNA was isolated from $50 \mathrm{ml}$ overnight culture using the QIAGEN Plasmid Midi Kit (Qiagen, Hilden, Germany) according to the manufacturer's instructions.

\section{BAC-library screening}

High-density colony filters of the libraries were prepared and screened by colony-hybridization as described [22]. The 'BC' library was also screened by the polymerase chain reaction (PCR) after isolating plasmid DNA from bacterial cells pooled at three levels: mini-pools, maxi-pools and super-pools. One thousand fifty six mini-pools were made from 96 clones each, 264 maxi-pools were prepared from 384 clones each (four mini-pools) and the 88 super-pools consisted of 1152 clones each (three maxi-pools). To identify a single positive clone, four rounds of PCR screening were performed. First, the DNA of the 88 super-pools was used as template. Second, the three maxi-pools constituting a positive super-pool were screened. Third, the four mini-pools of a positive maxi-pool were amplified and last, the 96 clones of the positive mini-pool were screened individually.

\section{Physical mapping}

Overlapping BACs were identified and ordered in two contigs corresponding to the two homologous chromo- 
somes of genotype P6/210 as described previously [22]. In short, BAC insertion ends were sequenced using T3 and T7 oligonucleotides as sequencing primers. The end sequences were used to detect overlaps, either based on $100 \%$ sequence identity with already sequenced BACs or by generating amplicons with identical sequences in different BACs. BAC contigs were assigned to either one or the other homologous chromosome by identifying DNA polymorphisms in insertion end sequences that were specific either for the allele inherited from parent P41 or parent $\mathrm{P} 40$.

\section{Genomic DNA Sequencing and Assembly}

Whole BAC clones were sequenced by the shotgun sequencing strategy. Custom sub-libraries of the BACs were prepared by GATC-Biotech AG (Konstanz, Germany). After physical fractionation of the BAC DNA, the random sheared fragments were blunt-ended by using T4 DNA-Polymerase and then ligated into the pCR4BluntTOPO vector (Invitrogen, California, USA). Approximately 1300 clones containing ca. $1.5 \mathrm{kbp}$ insertions and 300 to 400 clones with 4 to $5 \mathrm{kbp}$ insertions were produced for each BAC. The smaller inserts were amplified by colony-PCR using as primers TO2f (5'-agcggataacaatttcacacagga-3') and TO2r (5'-gacgttgtaaaacgacggccagtg-3'). The PCR was performed in a volume of $100 \mu \mathrm{l}$ containing $10 \mathrm{pmol}$ of each primer, $0.2 \mathrm{mM}$ dNTPs and $1.5 \mathrm{mM}$ $\mathrm{MgCl}_{2}, 0.2$ Units of Taq-Polymerase and the corresponding buffer from Invitrogen, California, USA. PCR conditions were: initial denaturation at $96^{\circ} \mathrm{C}$ for $5 \mathrm{~min}$ followed by 34 cycles of denaturation at $94^{\circ} \mathrm{C}$ for $50 \mathrm{sec}$, annealing at $55^{\circ} \mathrm{C}$ for $50 \mathrm{sec}$ and extension at $72^{\circ} \mathrm{C}$ for 3 min and a final extension for $4 \mathrm{~min}$ at $72^{\circ} \mathrm{C}$. Plasmid DNA was purified from the clones having $5 \mathrm{~kb}$ inserts using the BioRobot 9600 (Qiagen, Hilden, Germany). PCR products and plasmids were sequenced using T3 and T7 primers (Amersham, Pharmacia GE Healthcare BioSciences: Little Chalfont, UK). Sequencing reactions were performed by using the ABI PRISM BigDye Terminator Cycle Sequencing Ready Reaction Kit and an ABI377 automated DNA Sequencer (PE Biosystems, Foster City, California, USA). The approximately ten-fold redundant sequences were assembled using the PreGAP4 and GAP4 from Staden software package (Medical Research Council Laboratory of Molecular Biology, Cambridge, UK). The Lasergene software package (DNAstar, Madison, WI, USA) was used for sequence assemblies, comparisons and alignments. The sequences of overlapping BAC insertions were assembled after trimming any vector sequences using the SeqMan module of Lasergene and the Megamerger program, which is part of the EMBOSS package [52].

\section{Sequence analysis}

Dotter and MUMer [54] were used to align and compare genomic sequences. Putative exons and open reading frames (ORFs) were predicted by the programs GenMark.hmm [54], FGeneSH [55] and by alignment of EST (Expressed Sequence Tags) and protein sequences using GenomeThreader [56]. Genes were annotated by combining predicted ORFs from these gene finder programs with alignments of homologous sequences in public databases using the Apollo Genome Annotation Curation Tool [57] (Additional files 1 and 2). All genes were also manually annotated for putative function. Functional descriptions of homologous genes in the SWISSPROT database [58] were compared with homologous protein domains and patterns in the InterPro database [59]. Homologous genes in the SWISSPROT database were identified using BlastP [60] and homologous protein domains and patterns were identified by InterProScan [61]. Inparanoid [62] and BlastX [60] were used for the identification of similar genes in A. thaliana. The deduced amino acid sequences of the annotated ORFs were compared to A. thaliana annotated proteins from The A. thaliana Information Resource (TAIR) Release 6 [64]. The threshold criterion for accepting sequence similarity as significant was an E-value $<10^{-}$ 10 for BLASTP searches. Polyproteins and transposable elements were excluded from the comparison because of their limited information value. For the same reason, ORFs with more than 30 hits in the A. thaliana genome were also excluded [4]. A two-dimensional array was generated with the potato physical map of 420,000 bp in one dimension and the $\mathrm{A}$. thaliana physical map of $121 \mathrm{mb}$ in the other [4]. Hits of the putative potato proteins with $A$. thaliana annotated proteins were positioned in the array according to their base pair coordinates on the local potato and genome wide $A$. thaliana physical maps. Syntenic blocks were identified based on the criterion that at least three different ORFs within the potato contigs found hits within an A. thaliana genome fragment of similar size.

\section{List of abbreviations}

QTL, quantitative trait loci; QRL, quantitative resistance loci; ORF, open reading frame; RGL, resistance-gene-like; EST, Expressed Sequence Tags; BAC, bacterial artificial chromosome; PCR, polymerase chain reaction; $\mathrm{cM}$, Centimorgan; kbp, kilo base pairs; mb, mega base;

\section{Authors' contributions}

$\mathrm{AB}$ designed and conducted the experiments for constructing the physical map and BAC sequencing, did sequence annotation, synteny analysis and drafted the manuscript. HS, AJ and RB provided bioinformatics support (sequence analysis, sequence comparisons and ORF annotation). PV and BW contributed to BAC sequencing. HI, JP and KM constructed the BC-BAC library. CG and HS contributed substantially to manuscript preparation and editing. CG designed and oversaw the project. 


\section{Additional material}

\section{Additional File 1}

Figure S1: Gene annotation of R1-contig using APOLLO

Click here for file

[http://www.biomedcentral.com/content/supplementary/1471-

2164-8-112-S1.png]

\section{Additional File 2}

Figure S2: Gene annotation of r1-contig using APOLLO

Click here for file

[http://www.biomedcentral.com/content/supplementary/1471-

2164-8-112-S2.png]

\section{Additional File 3}

Figure S3: Dot-Plot comparison computed by MUMmer of the DNA sequence of the R1-contig and individual BAC sequences of S. demissum. One column represents one BAC sequence of $\mathrm{S}$. demissum. The first three columns (from left to right) are from haplotype A, the next seven from haplotype $B$ and the last two columns from haplotype $C$. The figure shows high similarity and co-linearity between the R1-contig and haplotype A of S. demissum. The first BAC (PGEC446J10, AC149228) is shown in reverse orientation. The region between 270 and $320 \mathrm{~kb}$ on the R1-contig is not covered by the non-overlapping BACs PGEC668E02 ( and PGEC472P22 (AC151815), so we can estimate the gap between these in haplotype A to be around $50 \mathrm{~kb}$ in size, covered by the unsequenced BAC PGEC597C19 according to Kuang et al. Only local and limited similarity and co-linearity is observed between the R1-contig and $B A C$ from the $B$ and $C$ haplotypes. The colour reflects percent sequence similarity, with red denoting close to $100 \%$ similarity.

Click here for file

[http://www.biomedcentral.com/content/supplementary/14712164-8-112-S3.doc]

\section{Additional File 4}

Figure S4: Dot-Plot comparison computed by MUMmer of the DNA sequence of the r1-contig and BAC sequences of $\mathrm{S}$. demissum. One column represents one BAC sequence of $\mathrm{S}$. demissum. The first three columns (from left to right) are BAC sequences from haplotype $A$, the next seven from haplotype $B$ and the last two columns from haplotype $C$. The figure shows two regions of high similarity and co-linearity between the r1-contig and haplotype B and $C$ of S. demissum, with similar but weaker similarity to haplotype A sequences. The first comprises the region between the beginning and $60 \mathrm{~kb}$ of the r1-contig and the second runs from $160 \mathrm{~kb}$ till the end of the r1-contig. The middle region shows some local similarity that is interrupted by non-conserved sequence. Most of the more significant similarities in this area reflect tandemly duplicated $R$ genes. The BAC sequences from haplotype $C$ do not contain this region, indicating a deletion in haplotype $C$ as found by Huang et al. An inverted repeat of a RNA-dependent $R N A$ polymerase leads to an X-shaped structure in the plot of the first $40 \mathrm{~kb}$ of the r1-contig against the S. demissum sequences, similar to the findings in Figure 2 when comparing to the R1contig (region $B$ ). The colour reflects percent sequence similarity, with red denoting close to $100 \%$ similarity.

Click here for file

[http://www.biomedcentral.com/content/supplementary/14712164-8-112-S4.doc]

\section{Additional File 5}

Table S1: Genomic sequence annotation of the R1- and r1-contig Click here for file

[http://www.biomedcentral.com/content/supplementary/14712164-8-112-S5.doc]

\section{Additional File 6}

Table S2: Putative orthologs from R1-, r1-contig and haplotypes A, B and $C$ of $\mathrm{S}$. demissum and the corresponding accession numbers are shown. Putative pseudogenes are indicated by "PS". The identities between transposons (TP) are not shown.

Click here for file

[http://www.biomedcentral.com/content/supplementary/14712164-8-112-S6.doc]

\section{Acknowledgements}

This research was funded under the GABI program (Genome analysis in the biological system of plants) by BMBF (Bundesministerium für Bildung und Forschung), project no 0312290 CONQUEST (Genes CONtrolling QUantitativE traits of Solanum Tuberosum). Part of this work was carried out in the department of plant breeding research and yield physiology, headed by Francesco Salamini, and in the department of plant breeding research and genetics, headed by Maarten Koornneef.

\section{References}

I. Gebhardt C: Potato Genetics: Molecular Maps and More in Biotechnology in Agriculture and Forestry. In Molecular Marker Systems Volume 55. Edited by: Lörz H, Wenzel G. Springer-Verlag, Berlin, Heidelberg; 2004:21 5-227.

2. Bonierable M, Plaisted RL, Tanksley SD: RFLP map based on a common set of clones reveal modes of chromosomal evolution in potato and tomato. Genetics 1988, 120:1095-I 103.

3. Gebhardt C, Ritter E, Debener T, Schachtschabel U, Walkemeier B, Uhrig U, Salamini F: RFLP analysis and linkage mapping in Solanum tuberosum. Theor Appl Genet 1989, 78:65-75.

4. Gebhardt C, Walkemeier B, Henselewski H, Barakat A, Delseny M, Stüber K: Comparative mapping between potato (Solanum tuberosum) and $A$. thaliana reveals structurally conserved domains and ancient duplications in the potato genome. The Plant J 2003, 34:529-54I.

5. Tanksley SD, Ganal MW, Prince JP, de Vicente MC, Bonierbale MW, Broun P, Fulton TM, Giovannoni JJ, Grandillo S, Martin GB, Messeguer R, Miller JC, Miller L, Paterson AH, Pineda O, Roeder MS, Wing RA, Wu W, Young ND: High density molecular linkage maps of the tomato and potato genomes. Genetics 1992, I32: I |4 I- I I60.

6. Gebhardt C, Valkonen JPT: Organization of genes controlling disease resistance in the potato genome. Annu Rev Phytopathol 200I, 39:79-102.

7. Meksem K, Leister D, Peleman J, Zabeau M, Salamini F, Gebhardt C: A high-resolution map of the vicinity of the $R I$ locus on chromosome $V$ of potato based on RFLP and AFLP markers. Mol Gen Genet 1995, 249:74-8I.

8. De Jong W, Forsyth A, Leister D, Gebhardt C, Baulcombe DC: A Potato hypersensitive resistance gene against potato virus $X$ maps to a resistance gene cluster on chromosome $\mathbf{V}$. Theor Appl Genet 1997, 5:153-162.

9. Ritter E, Debener T, Barone A, Salamini F, Gebhardt C: RFLP mapping on potato chromosomes of two genes controlling extreme resistance to potato virus X (PVX). Mol Gen Genet |99|, 227:8|-85.

10. Leonards-Schippers C, Gieffers W, Gebhardt C, Salamini F: The $\boldsymbol{R} \mathbf{I}$ gene conferring race-specific resistance to Phytophtora infestans in potato is located on potato chromosome V. Mol Gen Genet 1992, 233:278-283.

II. Leonards-Schippers C, Gieffers W, Schäfer-Pregl R, Ritter E, Knapp SJ, Salamini F, Gebhardt C: Quantitative resistance to Phytoph- 
thora infestans in potato: a case study for QTL mapping in an allogamous plant species. Genetics 1994, 137:67-77.

12. Oberhagemann $P$, Chatot-Balandras $C$, Bonnel E, Schäfer-Pregl $R$, Wegener D, Palomino C, Salamini F, Gebhardt C: A genetic analy sis of quantitative resistance to late blight in potato: Towards marker assisted selection. Mol Breed 1999, 5:399-4I5.

13. Collins A, Milbourne D, Ramsay L, Meyer R, Chatot-Balandras C, Oberhagemann P, De Jong W, Gebhardt C, Bonnel E, Waugh R: QTL for field resistance to late blight in potato are strongly correlated with earliness and vigour. Mol Breed 1999, 5:387-398.

14. Visker MHPW, Keizer LCP, Van Eck HJ, Jacobsen ELT, Colon LT, Struik PC: Can the QTL for late blight resistance on potato chromosome 5 be attributed to foliage maturity type? Theor Appl Genet 2003, 106:3 17-325.

15. Kreike CM, De Koning JRA, Vinke JH, Van Ooijen JW, Stiekema W]: Quantitatively-inherited resistance to Globodera pallida is dominated by one major locus in Solanum spegazzinii. Theor Appl Genet 1994, 88:764-769.

16. Rouppe van der Voort J, Wolters P, Folkertsma R, Hutten R, van Zandvoort P, Vinke H, Kanyuka K, Bendahmane A, Jacobsen E, Janssen R, Bakker J: Mapping of the cyst nematode resistance locus Gpa2 in potato using a strategy based on co-migrating AFLP markers. Theor Appl Genet 1997, 95:874-880.

17. Rouppe van der Voort J, van der Vossen E, Bakker E, Overmars H, van Zandvoort P, Hutten R, Klein-Lankhorst R, Bakker J: Two additive $Q T L s$ conferring broad-spectrum resistance in potato to Globodera pallida are localized on resistance gene clusters. Theor Appl Genet 2000, I0 I: I I 22-30.

18. Sattarzadeh A, Achenbach U, Lübeck J, Strahwald J, Tacke E, Hofferbert HR, Rotstein T, Gebhardt C: Single nucleotide polymorphism (SNP) genotyping as basis for developing a PCR-based marker highly diagnostic for potato varieties with high resistance to Globodera pallida pathotype Pa2/3. Mol Breed 2006, 4:30I-3I2.

19. Schäfer-Pregl R, Ritter E, Concilio L, Hesselbach J, Lovatti L, Walkemeier B, Thelen H, Salamini F, Gebhardt C: Analysis of quantitative trait loci (QTL) and quantitative trait alleles (QTA) for potato tuber yield and starch content. Theor Appl Genet 1998, 97:834-846.

20. Menendez CM, Ritter E, Schäfer-Pregl R, Walkemeier B, Kalde A Salamini F, Gebhardt C: Cold-sweetening in diploid potato. Mapping QTL and candidate genes. Genetics 2002, 162:1423-1434.

21. Bendahmane A, Querci M, Kanyuka K, Baulcombe DC: Agrobacterium transient expression system as a tool for the isolation of disease resistance genes: application to the $R \times 2$ locus in potato. Plant J 2000, 21:73-81.

22. Ballvora A, Ercolano MR, Weiss J, Meksem K, Bormann CA, Oberhagemann $P$, Salamini F, Gebhardt C: The RI gene for potato resistance to late blight (Phytophthora infestans) belongs to the leucine zipper/NBS/LRR class of plant resistance genes. The Plant J 2002, 30:36I-37I.

23. Chisholm ST, Coaker G, Day B, Staskawicz B]: Host-Microbe Interactions: Shaping the Evolution of the Plant Immune Response. Cell 2006, I24:803-8I4.

24. Ross H: Potato breeding. Problems and perspectives. In Adv Plant Breed Issue Supplement 13 Paul Parey Verlag, Berlin and Heidelberg; 1986.

25. Kuang H, Wei F, Marano MR, Wirtz U, Wang X, Liu J, Shum WP, Zaborsky J, Tallon LJ, Rensink W, Lobst S, Zhang P, Tornqvist CE, Tek A, Bamberg J, Helgeson J, Fry W, You F, Luo MC, Jiang J, Robin Buell C, Baker B: The $R I$ resistance gene cluster contains three groups of independently evolving, type $I R I$ homologues and shows substantial structural variation among haplotypes of Solanum demissum. The Plant J 2005, 44:37-5I.

26. Pryor $\mathrm{T}$, Ellis J: The genetic complexity of fungal resistance genes in plants. Adv Plant Pathol 1993, 10:28I-305.

27. Gebhardt C, Ballvora A, Walkemeier B, Oberhagemann P, Schüler K: Assessing genetic potential in germ plasm collections of crop plants by marker-trait association: a case study for potatoes with quantitative variation of resistance to late blight and maturity type. Mol Breed 2004, 13:93-102.

28. Salvi S, Tuberosa R: To clone or not to clone plant QTLs: present and future challenges. Trends Plant Sci 2005, 10:297-304.

29. Stein L: Genome annotation: From sequence to biology. Nature Reviews Genetics 200I, 2:493-503.
30. $\mathrm{Fu} \mathrm{H}$, Dooner HK: Intraspecific violation of genetic colinearity and its implications in maize. Proc Natl Acad Sci 2002, 99:9573-9578.

3I. Brunner S, Fengler K, Morgante M, Tingey S, Rafalski A: Evolution of DANN Sequence Nonhomologies among Maize Inbreds. Plant Cell 2005, 17:343-360.

32. Seah S, Yaghoobi J, Rossi M, Gleason CA, Williamson VM: The nematode gene, Mi- I, is associated with an inverted chromosomal segment in susceptible compared to resistant tomato. Theor Appl Genet 2004, 108: 1635-1642.

33. Barone A, Ritter E, Schachtschabel U, Debener T, Salamini F, Gebhardt C: Localization by restriction fragment length polymorphism mapping in potato of a major dominant gene conferring resistance to the potato cyst nematode Globodera rostochiensis. Mol Gen Genet 1990, 224:177-182.

34. Ganal MW, Tanksley SD: Recombination around Tm2a and Mi resistance genes in different crosses of Lycopersicom peruvianum. Theor Appl Genet 1996, 92:101-108.

35. Michelmore RW, Meyers BC: Clusters of resistance genes in plants evolve by divergent selection and a birth-and-death process. Genome Res 1998, 8:9828-9832.

36. Mao I, Begum D, Goff SA, Wing RA: Sequence and Analysis of the Tomato JOINTLESS Locus. Plant Physiol 200I, I 26(3): I33 I- I 340.

37. Rossberg M, Theres K, Acarkan A, Herrero R, Schmitt T, Schumacher K, Schmitz G, Schmidt R: Comparative sequence analysis reveals extensive microcolinearity in the Lateral Suppressor regions of the tomato, A. thaliana, and Capsella genomes. The Plant Cell 200I, I3:979-988.

38. The A. thaliana Genome Initiative: Analysis of the genome sequence of the flowering plant A. thaliana. Nature 2000 , 408:796-8I5.

39. The Rice Chromosome 3 Sequencing Consortium: Sequence, annotation, and analysis of synteny between rice chromosome 3 and diverged species. Genome 2006, 15:|284-|29|.

40. Panstruga R, Büschges R, Piffanelli P, Schulze-Lefert P: A contigous $60 \mathrm{~kb}$ genomic stretch from barley reveals molecular evidence for gene islands in a monocot genome. Nuc Acid Res 1998, 26:1056-1062.

4I. Hulbert SH, Webb CA, Smith SM, Sun Q: Resistance gene complexes: evolution and utilization. Annu Rev Phytopathol 200I, 39:285-3।2

42. Kepinski S, Leyser O: The A. thaliana F-box protein TIRI is an auxin receptor. Nature 2005, 435:446-45I.

43. Shirazu K, Schulze-Lefert P: Regulators of cell death in disease resistance. Plant Mol Biol 2000, 44:37I-385.

44. Schulze-Lefert P: Plant Immunity: The origami of receptor activation. Curr Biol 2004, I4:R22-R24.

45. Waterhause PM, Helliwell CA: Exploring plant genomes by RNA-induced gene silencing. Nat Rev Genet 2003, 4:29-38.

46. Ware $D$, Stein L: Comparison of genes among cereals. Curr $O P$ Plant Biol 2003, 6: I 2 I-I 27.

47. Bennetzen JL, Ma J: The genetic colinearity of rice and other cereals on the basis of genomic sequence analysis. Curr $O p$ Plant Biol 2003, 6: I 28-I33.

48. Ku H.-M, Vision T, Liu J, Tanksley SD: Comparing sequenced segments of the tomato and $A$. thaliana genomes: Large-scale duplication followed by selective gene loss creates a network of synteny. Proc Natl Acad Sci 2000, 97:912I-9126.

49. Dominguez I, Graziano E, Gebhardt C, Barakat A, Berry S, Arús P, Delseny M, Barnes S: Plant genome acheology: evidence for conserved ancestral chromosome segments in dicotyledonous plant species. Plant Biotech J 2003, I:91-99.

50. Kim UJ, Birren BW, Slepak T, Mancino V, Boyson C, Kang HL, Simon $\mathrm{MI}$, Shizuya $\mathrm{H}$ : Construction and characterization of a human bacterial artificial chromosome library. Genomics 1996 , 34:213-218.

5I. Meksem K, Zobrist K, Ruben E, Hyten D, Quanzhou T, Zhang H-B, Lightfoot DA: Two large-insert soybean genomic libraries constructed in a binary vector: applications in chromosome walking and genome wide physical mapping. Theor Appl Genet 2000, I0I:747-755.

52. Rice P, Longden I, Bleasby A: EMBOSS: the European Molecular Biology Open Software Suite. Trends Genet 2000, 16(6):276-7.

53. Sonnhammer EL, Durbin R: A dot-matrix program with dynamic threshold control suited for genomic DNA and protein sequence analysis. Gene 1995, 167:GCI-GCI0. 
54. Lukashin AV, Borodovsky M: GeneMark.hmm: new solutions for gene finding. Nuc Acids Res 1998, 26: I 107- I I I5.

55. Salamov AA, Solovyev VV: Ab initio Gene Finding in Drosophila Genomic DNA. Genome Res 2000, 10:516-522.

56. Gremme G, Brendel V, Sparks ME, Kurtz S: Engineering a software tool for gene structure prediction in higher organisms. Information and Software Technology 2005, 47:965-978.

57. Lewis SE, Searle SMJ, Harris N, Gibson M, lyer V, Ricter J, Wiel C, Bayraktaroglu L, Birney E, Crosby MA, Kaminker JS, Matthews B, Prochnik SE, Smith CD, Tupy JL, Rubin GM, Misra S, Mungall CJ, Clamp ME: Apollo: a sequence annotation editor. Genome Biol 2002, 3:research0082.

58. Bairoch A, Apweiler R: The SWISS-PROT protein sequence database and its supplement TrEMBL in 2000. Nuc Acids Res 2000, 28:45-48.

59. Apweiler R, Attwood TK, Bairoch A, Bateman A, Birney E, Biswas M, Bucher P, Cerutti L, Corpet F, Croning MDR, Durbin R, Falquet L, Fleishmann W, Gouzy J, Hermjakob H, Hulo N, Jonassen L, Kahn D, Kanapin A, Karavidopoulou Y, Lopez R, Marx B, Mulder NJ, Oinn TM, Pagni M, Servant P, Sigrist CJA, Zdobnov EM: The InterPro database, an integrated documentation resource for protein families, domains and functional sites. Bioinformatics 2000, 16:1145-1150.

60. Altschul F, Madden TL, Schaffer AA, Zhang J, Zhang Z, Miller W, Lipman DJ: Gapped BLAST and PSI-BLAST: a new generation of protein database search programs. Nuc Acids Res 1997, 25:3389-3402.

6I. Zdobnov EM, Apweiler R: InterProScan - an integration platform for the signature-recognition methods in InterPro. Bioinformatics 200I, I7:847-848.

62. Remm M, Storm CEV, Sonnhammer ELL: Automatic Clustering of Orthologs and In-paralogs from Pairwise Species Comparisons. JMB 200I, 14:104I-1052.

63. GABI Primary Database [https://gabi.rzpd.de/projects/Pomamo/ ]

64. The Arabidopsis Information Resource [http://www.arabidop sis.org]

Publish with Biomed Central and every scientist can read your work free of charge

"BioMed Central will be the most significant development for disseminating the results of biomedical research in our lifetime. "

Sir Paul Nurse, Cancer Research UK

Your research papers will be:

- available free of charge to the entire biomedical community

- peer reviewed and published immediately upon acceptance

- cited in PubMed and archived on PubMed Central

- yours - you keep the copyright

Submit your manuscript here:

http://www.biomedcentral.com/info/publishing_adv.asp
BiolMedcentral 